\title{
PHD THESIS SUMMARY: \\ Using case studies in the social sciences: methods, inferences, purposes
}

\author{
ATTILIA RUZZENE \\ PhD in philosophy and economics, November 2014 \\ EIPE, Erasmus University Rotterdam
}

Despite fads and fashions in the academic culture, case-based reasoning has proved to be a persistent form of analysis in the social sciences, in the humanities, and even in moral thinking. Broadly understood, casebased reasoning locates the ultimate source of our epistemic and moral intuitions in the concreteness and idiosyncrasy of particulars. Even though they can be traced back to a common root, different traditions of reasoning with cases and of using case studies coexist in the academic landscape. This thesis focuses primarily on the use of case studies in the social sciences as an epistemic strategy to formulate, establish, and generalize causal hypotheses. A secondary goal is an investigation into the use of causal findings generated within and by means of case studies to inform policy making in the social realm.

The thesis is organized in four chapters. In chapter 1, I characterize what can be regarded as two alternative views of case studies and the understanding of science in which they are embedded. The first approach flourished in the 1970s and looked at case studies as a special, and typically weaker, form of the experimental, statistical, or comparative methods. Since this approach tends to evaluate case studies by criteria belonging to other methodological traditions, it can be said to present a heteronomous paradigm. The second, alternative view, which was developed in the last decades, is taking shape gradually and is still far from being fully articulated. This approach strives for an understanding of case studies liberated from the narrow mindset that caricatures case studies as the method of last resort. In particular, it sees case studies as an autonomous epistemic genre (Morgan 2012).

In chapter 2, I address internal validity in historical narratives. Historical narratives are case studies that aim to formulate and substantiate causal hypotheses by articulating descriptions of the sequences of events leading to the outcome of interest. They typically 
make use of process-tracing to draw causal inference, and often rely on the additional use of the methods of comparison. Despite the important role of historical narratives in the social sciences, how process-tracing operates in the narratives is still poorly understood. The debate on process-tracing in fact, even though it is growing thanks to a number of recent contributions, is still muddy and under-developed. In particular, there are no shared criteria to assess its epistemic contribution; moreover, the conditions proposed so far tend to tie the validity of the findings to the use of specific kinds of evidence and are thus unhelpful when this specific evidence is not available.

I argue that the proposed conditions are unduly restrictive and fail to acknowledge the actual contributions process-tracing can offer to valid causal inference. I formulate new conditions to assess processtracing performance in cases in which the favourable evidential circumstances do not occur and existing criteria fail to apply.

In chapter 3, I address the problem of generalizability. I provide an outline of what I define as the traditional view on external validity. This approach is conditioned by a statistical viewpoint on case study research (CSR) and reduces external validity to issues of mere representativeness. In so doing it leads the debate on the generalizability of case-study results to a dead end as it quickly dismisses external validity as the downside of CSR. At the same time, it suggests that CSR is comparatively stronger in providing internally valid results. On this ground this approach recommends the use of case studies when internal validity is the main research goal of interest, while turning to other methods when one pursues generalizations instead. This outcome is unfortunate because, as a matter of fact, case studies are often performed with the explicit or implicit purpose of drawing lessons from the studied case to be carried over to new contexts yet unstudied.

I attempt to solve this tension by examining the assumptions behind the traditional view on the external validity of CSR. Some of these assumptions have already been addressed, and actually disputed, in the current debate. In chapter 3, I focus instead on those assumptions that, to the best of my knowledge, have not been addressed yet and seem to be responsible for the dead end in which the discussion among social scientists seems to be trapped now. In particular, I suggest that the debate should focus on how make case studies comparable rather than how select the typical case. Typicality and comparability are concepts 
closely related but distinct. The traditional view conflates the two and thus run into confusion about what external validity is really about and how it can actually be confronted in a fruitful manner. I surmise that by enhancing the comparability of studies unnoticed room for improvement is made for formulating more reliable assessment of the external validity of results obtained in case studies.

In chapter 4, I discuss issues of relevance when policy making purposes are at stake. In particular, I focus on the debate on the use and usefulness of randomized controlled trials (RCTs) to find the key to economic and social development. The participants to this debate agree that RCTs are affected by limited external validity, and that this impinges on their usefulness for policy making. They diverge, however, on the strategies to overcome this problem. I analyze three alternatives that are found in the economic literature: replication of RCTs, which has been proposed by the promoters of RCTs; cross-country regressions, which have been typically endorsed by RCT-sceptics; and the causal models proposed by James Heckman. I argue that these strategies succeed in their attempt to a different, and limited, extent.

Proponents of the first two strategies fail to take into adequate consideration the distinction between external validity and relevance, and treat the latter as a spill-over of the former. Their strategies, in fact, aim to improve the external validity of causal effects on the assumption that relevance will automatically follow. I argue that this is not the case because external validity and relevance are distinct concerns and should thus be confronted separately. The proposal by Heckman succeeds in delivering causal effects that are, as a matter of fact, more relevant to policy makers' purposes. I argue, however, that his model cannot adequately address the type of problems policy makers are likely to confront in developing contexts. Whereas Heckman's model is equipped to face problems of prediction, in developing contexts policy makers face problems of planning. Planning is a complex procedure that depends on various pieces of evidence and raises several concerns. Causal effects are but one epistemic input in this procedure; case-study evidence is also relevant to the crucial phases of planning.

\section{REFERENCES}

Morgan, Mary. 2012. Case studies: one observation or many? Justification or discovery? Philosophy of Science, 79 (5): 667-677. 
Attilia Ruzzene is a lecturer at the Faculty of Philosophy of Erasmus University Rotterdam. She studied international and diplomatic science at the University of Torino where she obtained an MSc (2006) and a PhD (2010) in economics. In the meantime she developed an interest in the philosophy and methodology of the social sciences and moved to Erasmus University Rotterdam. At the Erasmus Institute for Philosophy and Economics (EIPE), she obtained a research master in philosophy of economics (2009) and embarked on her second $\mathrm{PhD}$ project under the supervision of Julian Reiss and Jack Vromen.

Contact e-mail: <ruzzene@fwb.eur.nl> 